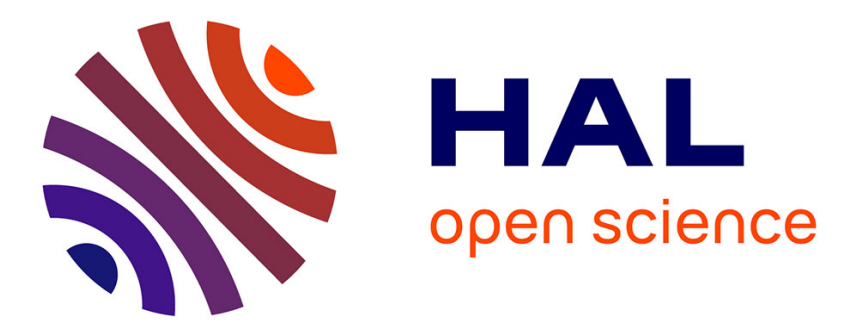

\title{
Mobile Crowd-Sensing as a Resource for Contextualized Urban Public Policies: A Study using Three Use Cases on Noise and Soundscape Monitoring
}

\author{
Bruno Lefèvre, Rachit Agarwal, Valérie Issarny, Vivien Mallet
}

\section{To cite this version:}

Bruno Lefèvre, Rachit Agarwal, Valérie Issarny, Vivien Mallet. Mobile Crowd-Sensing as a Resource for Contextualized Urban Public Policies: A Study using Three Use Cases on Noise and Soundscape Monitoring. Cities \& Health, 2019, 10.1080/23748834.2019.1617656 . hal-02127052v2

HAL Id: hal-02127052

https://hal.inria.fr/hal-02127052v2

Submitted on 27 May 2019

HAL is a multi-disciplinary open access archive for the deposit and dissemination of scientific research documents, whether they are published or not. The documents may come from teaching and research institutions in France or abroad, or from public or private research centers.
L'archive ouverte pluridisciplinaire HAL, est destinée au dépôt et à la diffusion de documents scientifiques de niveau recherche, publiés ou non, émanant des établissements d'enseignement et de recherche français ou étrangers, des laboratoires publics ou privés. 


\section{Mobile Crowd-Sensing as a Resource for Contextualized Urban Public Policies: A Study using Three Use Cases on Noise and Soundscape Monitoring}

5 Bruno Lefevre $^{\mathrm{a}}$, Rachit Agarwal ${ }^{\mathrm{a}}$, Valerie Issarny ${ }^{\mathrm{a}}$, Vivien Mallet $^{\mathrm{a}}$

${ }^{a}$ Inria Paris, 2 Rue Simone Iff, Paris, France

\section{ARTICLE HISTORY}

Compiled May 27, 2019

\section{ABSTRACT}

Environmental noise is a major pollutant in contemporary cities and calls for the active monitoring of noise levels to spot the locations where it most affects the people's health and well-being. However, due to the complex relationship between environmental noise and its perception by the citizens, it is not sufficient to quantitatively measure environmental noise. We need to collect and aggregate contextualized -both quantitative and qualitative- data about the urban environmental noise so as to be able to study the objective and subjective relationships between sound and living beings. This complex knowledge is a prerequisite for making efficient territorial public policies for soundscapes that are inclined towards living beings welfare. In this paper, we investigate how Mobile Phone Sensing (MPS) - aka crowdsensing- enables the gathering of such knowledge, provided the implementation of sensing protocols that are customized according to the context of use and the intended exploitation of the data. Through three case studies that we carried out in France and Finland, we show that MPS is not solely a tool that contributes to sensitizing citizens and decision-makers about noise pollution; it also contributes to increasing our knowledge about the impact of the environmental noise on people's health and well-being in relation to its physical and subjective perception.

\section{KEYWORDS}

Environmental Noise, Urban Health, Well-being, Crowd-sensing, Mobile Phone Sensing, Noise maps, Ambiciti, Soundscape

\section{Introduction}

Noise is a pollutant that raises significant health and environmental issues, especially in cities. According to the World Health Organization (WHO, 2018): "one in five Europeans is regularly exposed to sound levels at night that could significantly damage health", and in high-income European countries, "the noise attendant health risks account for a loss of 1 - 1.6 million Disability Adjusted Life Years (DALYs)" due to illness, disability or early death. The WHO guidelines set environmental noise exposure limits in various environments with regards to major health concerns (WHO, 2018): hearing and cognitive impairments, cardiovascular and biological diseases, annoyance, effects on sleep, and more globally a decreasing quality of life and well-being. Nevertheless, from an epidemiological perspective, we still know little about the spe- 


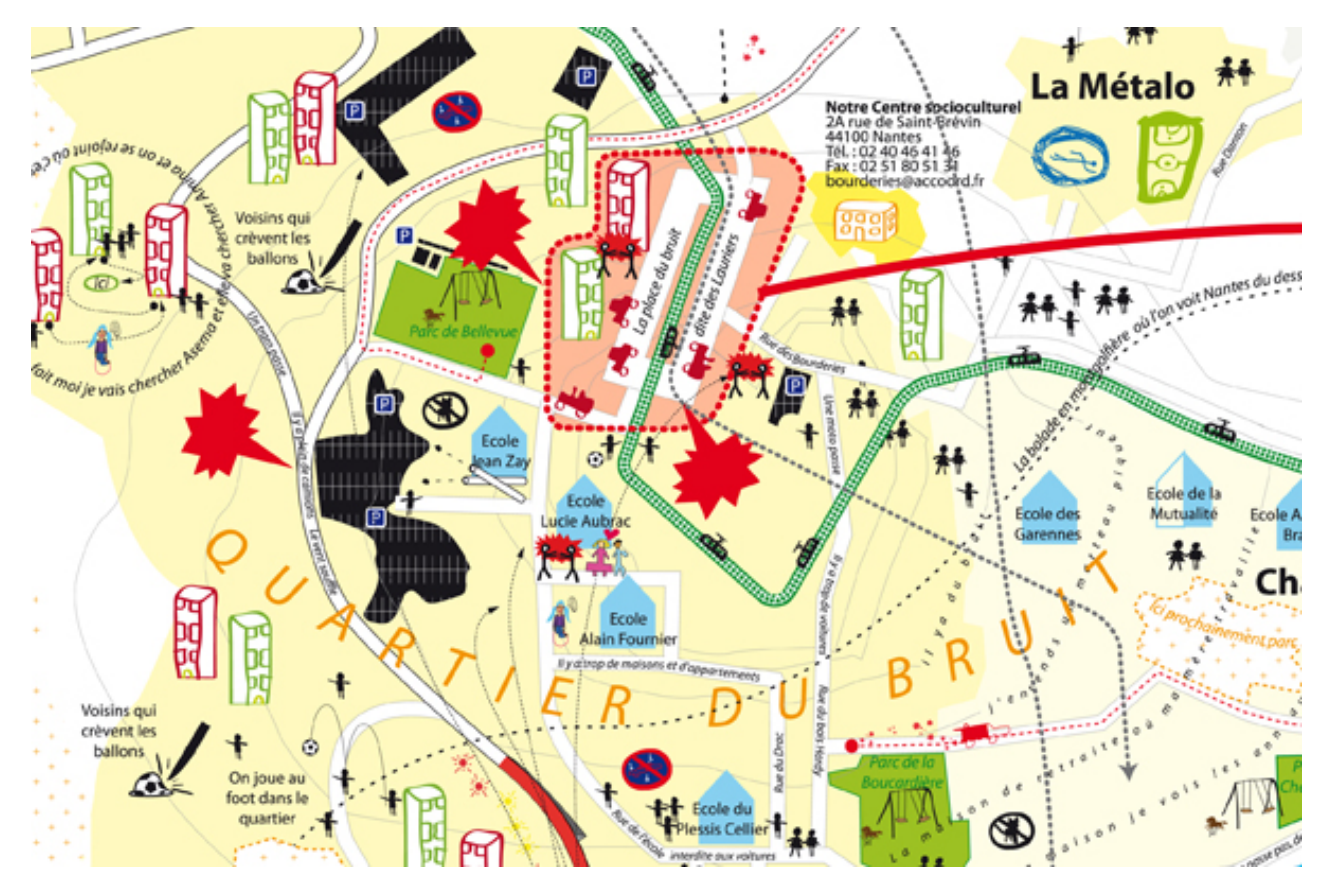

Figure 1. A sensitive map of a district in the city of Nantes, France, 2013 (Jourdan, 2013).

cific causal relationships between the citizens' exposure to noise and their physiological and psychological health (Fu et al., 2017; Munzel, Gori, Babisch, \& Basner, 2014). The evaluation of the impact of the exposure to noise on health is primarily self-reported and influenced by more global psychological conditions of individuals as well as by

45 the context of each perception experience. For instance, noise-sensitive people tend to report more neurological and emotional symptoms than others (Kumar et al., 2017; Stansfeld \& Shipley, 2015). Loud music can provide a sense of well-being during a concert. Previous study has notably shown a great variability between the measured and the perceived noise at low intensity - under $57 \mathrm{~dB}(\mathrm{~A})$ - (Baranzini, Schaerer, Ramirez,

50 \& Thalmann, 2010; Seixas, Daniell, Neitzel, Sheppard, \& Davies, 2008). The above points out the need for combining the individual and collective monitoring of the effects of noise on health and well-being, while taking into account the social context in which diseases are identified.

Further, we need to consider the contribution of noise to the urban soundscapes 55 that shape one's relationship with other citizens and the city. A soundscape is an "acoustic environment as perceived or experienced and/or understood by a person or people, in context" (Kornprobst, Manvell, Cook, \& Gigant, 2014; Zannin, Calixto, Diniz, \& Ferreira, 2003; Zhang, Ba, Kang, \& Meng, 2018), and is therefore defined as "the complex superposition of natural, human, and technical noises" (Genuit \& Fiebig,

60 2006). As an illustration, "Emotional cartographers" (Olmedo, 2015) or artists have produced subjective sensitive city maps (see Figure 1), which recognize sound and noise as fundamental and intimate dimensions of the relationship between the citizens and the urban space (Marry, 2011). More recently, urban soundscape designers and noise architects are integrating environmental noise in the planning strategies 65 of public spaces (Meng \& Kang, 2016; Sanchez, Alves, \& Botteldooren, 2018). The soundscapes are constitutive of the urban environment; they inform citizens about their surroundings, who may adapt their behavior accordingly. However, the subjec- 


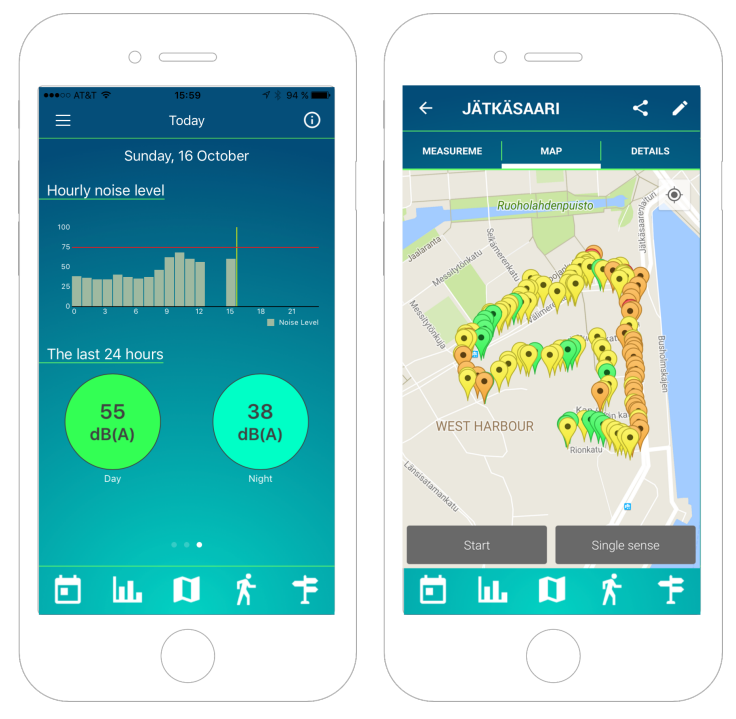

Figure 2. Screen shots of the Ambiciti app. The individual exposure to noise is provided daily, weekly or monthly, and alerts of potential health risks (left). The Journey mode allows the users to monitor their exposure to noise along specific paths (right).

tive and contextualized dimensions of soundscapes challenge the analysis of specific observations about the perception of environmental noise and its impact on citizens' urban noise to better understand its effects on urban dwellers' health and well-being. Soundscapes must be considered as contexts that are culturally and socially framed. This requires observing the relationships between noise and people with reference to specific contexts, that is, monitoring the physical characteristics of the noise together with its contextualized perception.

In this paper, we show that Mobile Phone Sensing -MPS aka crowdsensing- is a promising technology to address the above. On the one hand, mobile phones embed noise sensors -the microphones- and systems that may serve monitoring the physical properties of an acoustic vibration, which are measurable by its intensity, frequency or wave length (Lane et al., 2010; Maisonneuve, Stevens, \& Ochab, 2016; Ventura, Mallet, Issarny, Raverdy, \& Rebhi, 2017). On the other hand, mobile apps may serve gathering the end-user's subjective and contextualized perception of noise, that is, its psycho-acoustical characteristics (Jambrošić, 2017; Zwicker \& Fastl, 2007). Our research specifically leverages the Ambiciti crowdsensing app, and associated cloudbased platform for data aggregation, which we developed at Inria in collaboration with private partners (Hachem et al., 2015; Issarny et al., 2016; Lefevre \& Issarny, 2018). The Ambiciti app has been available for both iOS and Android devices since 2016. Among other features, the app monitors the personal exposure to noise measured in $\mathrm{dB}(\mathrm{A})$ (see Figure 2). The default app settings provide average noise levels from 90 automatic 5 second-long measurements every 5 (Android) or 7.5 (iOS) minutes, while the user may change the duration and frequency of the measurements in the parameter section. The heterogeneity of the embedded sensors requires the calibration of the app so as to ensure the sufficient accuracy of the reported measurements. We analyzed that the bias for the phones of the same model is almost identical (Ventura et al., 95 2017). This leads us to maintain the known biases in an Ambiciti database so that they can be reused as default bias values as far as possible. Still, the per-device manual 
calibration using a professional sound-level meter is the most effective approach, for which the app offers a dedicated function.

Thanks to the experiments carried out using Ambiciti from 2016 to 2018, this paper surveys the relevance of MPS for the monitoring of noise and soundscapes, provided the design of the supporting observation protocol in relation with the target social context. The paper contributions decompose as follows:

(1) First, we review the key challenges facing a better knowledge of the relationship between environmental noise, its measurement and its perception by citizens. This allows us to analyze the relevance (and limits) of MPS to address these requirements (Section 2).

(2) Second, we introduce the MPS protocols that we designed in relation with three specific use cases. The use cases are all concerned with a better understanding of the impact of noise on health and/or well-being, but they differ with respect to their soundscapes within the following respective contexts: city-wide, zoo, and school. The associated experiments enable us to analyze the technical and societal contributions of MPS toward informing our knowledge of the population exposure to noise pollution. We show in particular the following added-value of MPS: (i) It provisions more representative data and noise maps (Section 3); (ii) It allows correlating noise observations to physiological and social behavior monitoring, especially if applied over a long time period in spatially insulated spaces (Section 4); (iii) It contributes to jointly monitor technically measured and perceived noise environments (Section 5).

(3) Finally, we conclude by highlighting the relevance of MPS as a tool to inform decision-makers toward eliciting policies that combine health protection, and increase welfare and territorial sustainability. Such a participatory technology further contributes to increasing the awareness of environmental noise across the overall population, beyond the decision-makers.

\section{MPS for more contextualized policies}

125 Although noise pollution is known to affect the individual's health and well-being, its reduction in the urban environment remains a challenge for local decision-makers. Indeed, noise has a dual, somehow conflicting, impact on the population. On the one hand, the exposure to excessive noise affects the citizens' health: numerous acoustical, physiological, and psychological troubles are due to the short term exposures to high levels of noise, as well as to the long term exposures to stressful environmental noises. On the other hand, the soundscapes contribute to the citizens' social relationships and well-being -notably in occupational and leisure contexts- since noise also provides acoustical information about our dynamical and spatial environment.

\subsection{Contextualized noise policies and social practices}

Since the early 2000s, European and International institutions have engaged in proactive scientific and legal noise programs (Unit, 2017). Their intent is to develop a better knowledge about noise emission sources, and to encourage governing bodies to act toward reducing the citizens' exposure to urban noise. For instance, European cities with more than 100k inhabitants are legally bound to provide relevant information about their environmental noise. The European 2002 Directive (Official Journal of the 
European Communities, 2002) aims to support national and local strategies to reduce noise pollution and to minimize the citizens' exposure. However, the European Commission (The Centre for Strategy \& Evaluation Services and ACCON, 2016) stresses "significant differences" between the effective "less or more centralized" execution of this directive in the member states. In practice, only a few countries and cities have pro-actively implemented noise-exposure reduction strategies, except when related to major risk sites such as industrial areas (Manwar, Mandal, \& Pal, 2016), airports, and high traffic zones. In 2013, only $44 \%$ of the concerned cities had effectively delivered such expected data about their noise environment (Blanes et al., 2017). Overall, "considerable delays" have been observed for the production of "strategic noise maps and environmental noise prevention plans" in all the countries. Two main reasons explain the delay. First, in spite of a growing awareness and actual efforts of policy-makers, environmental noise remains poorly integrated in urban planning strategies. Second, the noise monitoring technologies that are available to technical services in local institutions (mainly data modeling) do not always provide accurate and dynamic-enough information to relate noise environments, their perception, and health concerns.

We argue that a better inclusion of noise and soundscapes in local policy making requires:

(1) Characterizing precisely the required noise information in relation with the political and societal ambition, since noise reduction may impact issues as diverse as health, well-being, urban attractiveness, the functioning of the city;

(2) Solving the technological issues arising for the production of accurate and relevant knowledge about urban environmental noise. The issues at stake relate to the supporting sensors, data analytics, noise mapping and forecasting, together with the ability to collect the urban dwellers' perception and well-being feelings;

(3) Managing the internal organization -spanning the city departments of urban planning, environment and sustainable development, economy, innovation- and institutional partnerships given the shared competences from the local to the international levels;

(4) Encouraging the development of new social practices such as: participatory governance, increasing sensitivity to environmental issues, trustful uses of IT and personal data exploitation.

Existing studies stress "critical data and research gaps to be addressed in future studies" (Jarosinska et al., 2018) to improve our knowledge of the relationships between urban noise and health. In particular, noise maps with high resolution (under 15 meters) and offering average or dynamic representations of the city in A-weighted decibels $(\mathrm{dB}(\mathrm{A}))$ values would be very helpful to decision-makers (Unit, 2016). Such original resources can contribute to a better understanding of the complex and changing effects of urban noise on the citizens' health and well-being. In this paper, we

180 advocate that Mobile Phone Sensing (MPS) is a promising technology to support the above.

\subsection{MPS for a contributive characterization of urban soundscapes}

In the urban areas of developed countries, more than $80 \%$ of the population owns a smartphone. Worldwide, this ratio decreases to $68 \%$ of the global population. Among them, as of 2017, $56 \%$ use a $4 \mathrm{G}$ connection (Deloite, 2017). In 2018, the smartphone users downloaded 205.4 billions apps worldwide (Statistics, 2018) with an average use of 40 apps per month (AppAnnie, 2018). The massive adoption of smartphones 
-and related apps- together with the increasing coverage of wireless networking create a significant opportunity to involve people in contributing -quantitative as much as qualitative - data about the urban environment (Hachem et al., 2015). In particular, smartphone users are potential producers of original and contextualized quantitative information about the environment in which they live, thanks to the increasing capacities of the sensors embedded in their smartphones. However, the contributed information requires the use of advanced filtering and interpolation tools so as to ensure that the provided observations are accurate enough for inclusion in the mapping of urban noise (Ventura, Mallet, \& Issarny, 2018).

Although there are socio-economical and cultural disparities worldwide (Cho, 2015), MPS has the potential to contribute to the collection of information about environmental noise at a very large scale -across space and time. Such participatory sensing 200 of noise levels by citizens results from the availability of the relevant technological resources as much as from social practices (Goncalves, Hosio, Rogstadius, Karapanos, \& Kostakos, 2015). Indeed, MPS is not only fueled by increasingly more powerful devices and networks, but also by a growing awareness about noise and environmental issues. People leverage available collaborative technologies to contribute new knowledge about 205 the urban environment and ultimately engage in collective actions toward its improvement. Still, the specific context of measurements using MPS must be considered, which depends on the motivations of the contributing users, the attention given to the action, and the users' expectation about the exploitation of the MPS data. In other words, users are not merely passive activators of the predefined technical features of a given

210 MPS app. MPS users actively engage in a complex process that is partly configured by the features and interfaces of the app, and partly by subjective and contextual parameters (Agostinelli, 2009). The social environment in which the participating users are at the specific moment -alone or in a group, for professional or personal concerns, out of curiosity or to get pieces of evidence of a noise environment- contributes to the 215 way they engage in the MPS-based process. The users' IT skill and trust in privacy protection (Noureen \& Asif, 2017) are other criteria that influence the sensing process. Noise or soundscapes sensing must thus be acknowledged as an experience (Bonfils, 2015). Using a MPS app is a communicational arrangement (Miège, 2007): a user drives an abduction (Dorst, 2015) mental process to engage in the act of making a noise measurement. The user's previous experiences of using apps and knowledge of mobile noise measurement lead to specific assumptions about the measurement process (e.g., How to handle the phone? How long a measurement should last?) and the meaning of the data communicated by the app (e.g., average levels, continuous levels or peaks do not provide the same kind of information). Even if the user interface of the app provides a rationally predefined interaction path, each measurement session is a specific and subjective process in which the user engages. This process implements trade-offs between the information that the user expects to obtain from the collected data and the operational efficiency of the data collection in the current situation.

Due to the subjectivity of the sensing experience, MPS data must be contextual230 ized to provide meaningful information (Issarny et al., 2016). Knowing the context also serves in the processing of the aggregation (aka assimilation) of data provided by a large population of users and by other sources (such as simulation results and high-end sensors). Mathematical algorithms for error corrections, filtering, weighting, spatial and temporal interpolations enable compensating the low reliability of part of

235 the MPS data. Typically, MPS-based noise measurements are of significance only if the microphone -like other sensors- is calibrated (i.e., the bias is known) and unobstructed (e.g., this may be estimated using the proximity sensor that must be "off"). We also 
need to have accurate information about the location (i.e., known using GPS and/or the network) of any collected noise level data to enable further analysis. Additional contextual parameters contribute to make the collected data more significant, among which: the situation (in-/out-door, under-/above-ground), the mobility mode (walking, cycling, driving, in public transports), the qualitative nature of the noise source. However, the mobile phone and its embedded sensors allow inferring only partially such relevant contextual information. The active contribution of the users, influenced by their previous experiences or guidelines, is critical to ensure the relevance of the crowd-sensed data.

MPS empowers citizens to become active contributors to environmental and urban planning policies, and to a new form of knowledge about their living space. Noise and soundscapes monitoring using MPS involves collective dynamics that aim at bringing to the forefront new environmental issues that must be taken into account in the management of the city. Indeed, a noise map provides a representation of a city or of an urban area. That image can reinforce or contradict the symbolic and material identity of a territory. Whether positively or negatively, it affects the image of a territory for its citizens with regards to other competing territories. Making materially visible -

255 through quantitative data and visual representations- a dimension of the city that was previously only subjectively felt contributes to changing this living area as well as its relationships with citizens.

\subsection{MPS as an opportunity to enhance our knowledge of the acoustic environment}

MPS technologies can contribute to conceive locally-anchored and relevant strategies for a better understanding and management of urban noise. However, the social practices and motivations of the MPS users impact directly upon the quantity and quality of the noise data that is gathered. Specific and formally supported initiatives focused on noise sensing can increase the sensitization and involvement of urban citizens. Still, the characterization of the urban noise environment benefits from the aggregation of complementary datasets that are generated using a combination of technologies (spanning MPS, high-end sensors, simulation). The collection of noise measurements using MPS is one such data source, provided the users gather the data in the appropriate context.

270 We identify three main opportunities that MPS brings to a better contextualized correlation of acoustic exposure with health and well-being:

(1) The most evident contribution of MPS is to help provide an accurate and dynamic representation of the urban soundscape that takes into account the actual human activity over time. Indeed, existing simulated maps and using high-end sensors provide only partial information about it. However, solving this issue implies gathering a critical mass of sufficiently accurate measurements across space and time, which is a challenge;

(2) The low economic, technical and practical costs of MPS favors its adoption in various specific local strategies. For instance, with the help of supporting protocols, MPS provides an easily accessible tool to monitor the effects of particular noisy activities such as site construction. Further, MPS facilitates the correlation of soundscapes with health or well-being effects, either simultaneously (through the gathering of relevant information from the user's direct input and/or connected physiological sensors) or via the post-analysis of the collected data; 
(3) Thanks to the involvement of individuals in noise measurements, MPS makes possible the "sensing" of the subjective perception of noise while its intensity is technically measured. The collection of individual noise levels measurements and perceptions can then provide original resources for a better epidemiological understanding of noise effects.

The following sections report on the empirical study of the MPS contributions to the above opportunities. We have specifically run three supporting experiments, from 2016 and 2018, in France and Finland. The experiments highlight the customization of the MPS protocols that we implemented according to the specific aim of what is to be measured, monitored and related, but also the organizational/social context (who is concerned, who is driving the experiment). The customization then allows the evaluation of soundscapes in relation with their reception by living forms, including physiological and psychological conditions.

\section{MPS for a more accurate and temporal representation of urban soundscapes}

A noisy environment results from the interaction between noise-producing activities (emitted within the urban territory or imported) and characteristics of the city that are both structural and atmospheric. Urban noise is also intimately related to a wide range of one-time or recurrent human activities. This changing characteristic of soundscapes makes challenging its estimation at a scale that is significant enough to allow its correlation with health concerns. Providing relevant and dynamic information about the surrounding noise levels may then benefit from MPS to grasp the temporal dynamics of the urban soundscapes. Still, noise mapping at the urban scale requires leveraging MPS in combination with state of the art approaches, that is, mathematical modeling and using accurate professional sensors.

${ }^{1}$ https://www.soundplan.eu/english/soundplan-acoustics/, accessed 03/19.

${ }^{2}$ https://www.datakustik.com/products/cadnaa/cadnaa/, accessed 03/19.

${ }^{3}$ https://github.com/Ifsttar/NoiseModelling/wiki/01-Installation, accessed 03/19. 


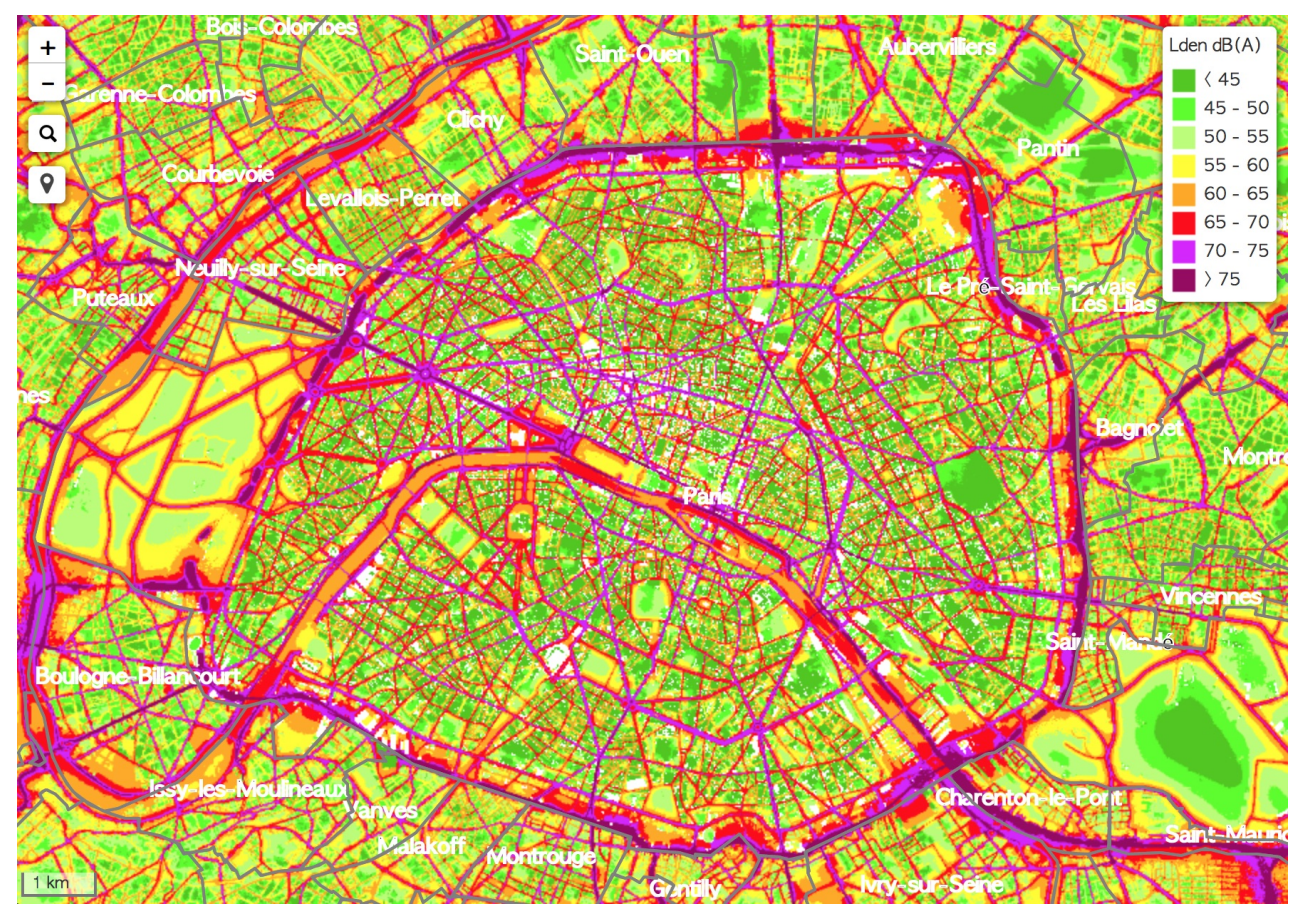

Figure 3. A modeled transportation-based noise map of Paris city, France. Average data for day, evening and night. [Source: Agence d'Écologie Urbaine de la ville de Paris, Direction des espaces verts et de l'environnement, 2017.]

like noisy events related to occasional and recurring human activities. Interpolation techniques tend to smooth noise levels and hide trouble spots that are apparent at a fine scale mapping. Although models can help us to better understand global trends in urban territories, they can also show shortcomings for an accurate and dynamic description of the citizens' exposure to noise.

To deal with the limitations of mathematical models, high-quality noise sensors are now used in cities, mainly close to "sensitive" sites (e.g., airports, industrial areas, high traffic axes). These sensors have a spatially limited coverage and are expensive. However, thanks to their high precision and reliability, the gathered observations are very useful in minimizing the inaccuracies of the mathematical models. Integrating and interpolating the observed high-quality data allows enhanced accuracy of the whole urban modeling (Aumond et al., 2017).

Still, MPS appears as a promising complementary technology to the above considering the massive use of smartphones that embed increasingly accurate microphones (Kardous \& Shaw, 2015) and location systems (Longo, Zappatore, Bochicchio, \& Navathe, 2017). In recent years, and notably since most cities have engaged in smart projects, many studies have pointed out the opportunities and issues of MPS contributions for a better knowledge of the urban environment. On the negative side, the smartphone microphones remain -relatively- low-quality noise sensors and provide only rough values about the actual surrounding context. Nevertheless, their calibration and bias correction improve the reliability of the data that MPS can produce (Ventura et al., 2017). Furthermore, the location sensors, via GPS or mobile networks, enable reaching very high spatial resolutions (from 3 to 5 meters) on most of the city area. However, where the smartphone and microphone are (including how and where held and positioned) greatly conditions the accuracy of the location and of the noise 

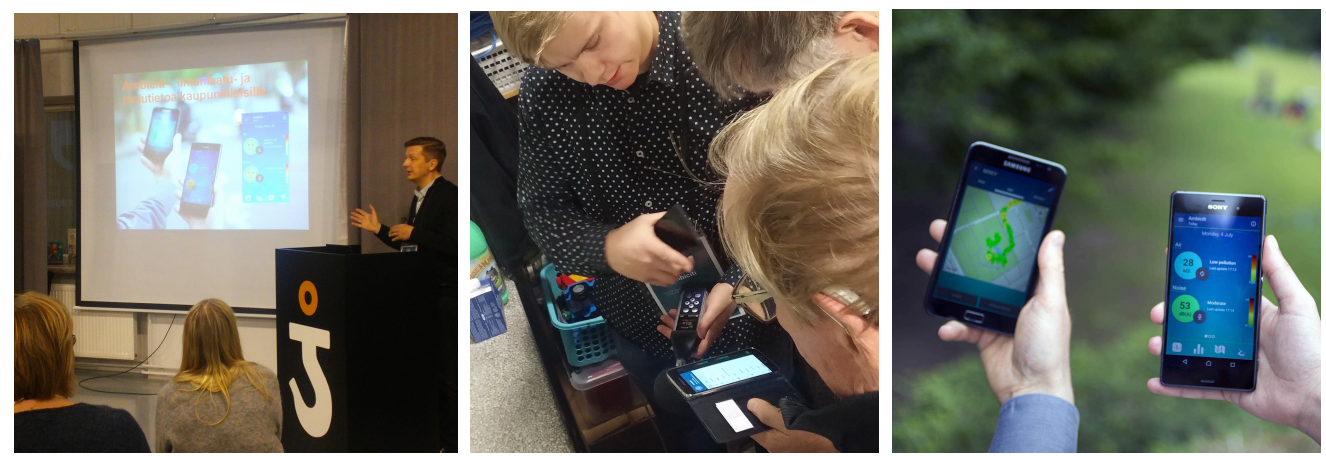

Figure 4. An Ambiciti workshop: (a) MPS presentation, (b) Calibration of the participants' phones, and (c) Measurement session through a walking trip in a nearby district.

measurements. Henceforth, a critical challenge is to reach a critical mass of MPS contributions, engaging a sufficient crowd of users who use the MPS app in a proper way. Indeed, gathering a large amount of reliable data is a condition to adequately cover the overall urban area -across time and space- to correct biases and to homogenize and interpolate data. A follow-up challenge is the ability to collect and analyze the crowd-sensed data at scale.

When combined, and with the help of data assimilation algorithms (Ventura et al., 2018), the above technologies - mathematical modeling, high-quality sensing, MPSprovide more reliable and time-based representations of the urban noise. By improving the mathematical models and interpolating measurements, noise crowd-sensing contributes to enhance the forecasting of the urban noise environment.

\subsection{MPS protocol to contribute to the urban noise mapping}

To validate the above claim about the added value of MPS to urban noise mapping, we first devised a supporting MPS protocol, which we assessed through dedicated experiments in France, Belgium and Finland. One related aim is to contribute to the study of the correlation between soundscapes and health concerns, which ultimately requires complementary epidemiological health data.

We structured the experiments around Ambiciti sensitization workshops, for which we locally issued calls for participation with the support of a local organization or institution (e.g., Mission ville intelligente in Paris and Forum Virium in Helsinki). A workshop typically lasts about 2 hours and involves a dozen of participants.

A workshop begins with an introduction to the impact of noise pollution on public health and to Ambiciti's main features (see Figure 4(a)). Then the participants' phones are calibrated -as need be- using a professional Sound-Level Meter (SLM), where successive 20 second long measurements of a pink noise streamed by loudspeakers are made simultaneously by the SLM and the user's mobile (see Figure 4(b)). The comparison of the measured levels provides indication about the bias of the mobile microphone, in $\mathrm{dB}(\mathrm{A})$. Once all the phones are calibrated, the participants engage in a "Ambiciti-MPS measurement session". That is, the participants collectively and pro-actively collect noise observations in a chosen nearby district by walking during 20 minutes along the same route (see Figure 4(c)). The data collection leverages the "Journey" mode of the Ambiciti app (see Figure 5), which ensures continuous 5 secondlong measurements and involves a proactive user contribution as opposed to the default 

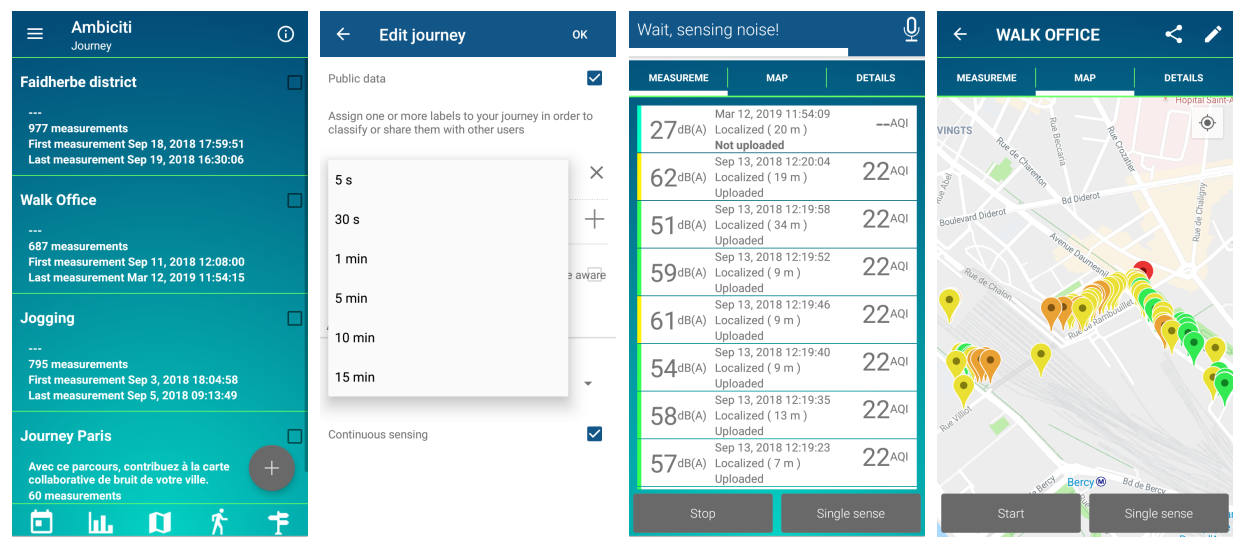

Figure 5. The Journey mode of the Ambiciti app showcasing: (a) Creation of a "Journey", (b) Edition of the measurement settings, including the sampling interval and duration, each set to 5 seconds by default, (c) Listing of the measured noise levels along with associated location accuracy and other information, and $(d)$ Display of the measured noise levels on a map.

passive measurements in the background.

Each user generates about 200 individual noise levels measurements along the route. All the participants share their own measurements, which are collected at the Ambiciti server. The server then processes (through filtering, assimilating, interpolating) the data to build a collective, optimized noise map of the walked area. Finally, the resulting enhanced noise map of the area (on the basis of the filtered and assimilated data) is presented so that the participants may witness and exchange about the contribution of their collective data sensing. As part of the Ambiciti workshops, we interviewed individual users, representatives of local civil organizations, and members of institutional bodies. Our aim was to get feedback about the actual relevance of MPS in specific situations and to evaluate the political, institutional and socio-economical characteristics of the local contexts. In particular, the interviews focused on the institutional awareness of the impacts of noise on urban dwellers and on the challenges in carrying out efficient strategies concerning soundscapes for enhancing well-being.

\subsection{Assessing the MPS contribution to representative and fine scale soundscapes: Experiments in Paris}

400 Among the workshops that we organized, we held two in Paris in 2016: one during the inauguration of "La Canopée" in Les Halles district, and the other during the Digital Science Festival "Futur en Seine" in the République district. Figure 6 shows the resulting optimized maps compared to the initial simulated maps. The MPS reveals that the modeled noise levels are significantly under-estimated, probably because of anthropogenic sources of noise, which the models do not take into account (Ventura et al., 2018). The actual activity in cities generates noises that can not be modeled because of their wide variety, high fluctuation and unpredictability. MPS can, therefore, contribute to minimize the errors of the models and to provide a more accurate representation of the actual exposure to noise at a specific moment.

In addition to the organization of sensitization workshops about MPS-based noise measurements, we have investigated the relevance of MPS to improve the global representativeness of noise maps for an entire city. Throughout the year 2017, we collected about 2.43 million noise measurements from the Ambiciti users, which are localized 

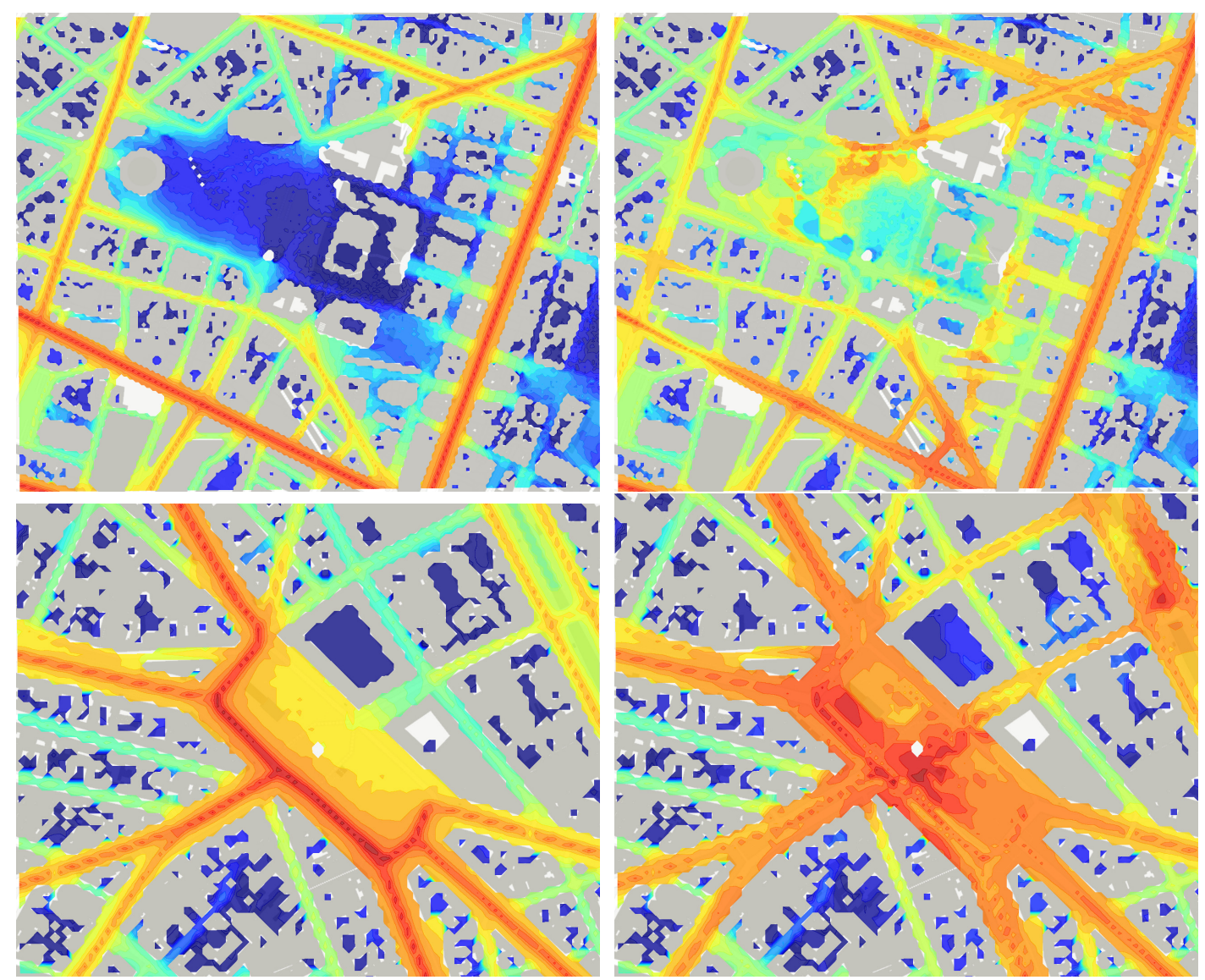

Figure 6. Noisemaps of the Canopée des Halles (top) and Place de la République (bottom), in Paris (France), without (left) and with (right) interpolated crowd-sensed data, collected via Ambiciti on April, 9th, 2016 and June, 11th, 2016 (Ventura et al., 2018).

in Paris (See Table 1). Most of the measurements come from the default automatic journey mode). We filtered this raw data according to its reliability and relevance, to provide more representative information about the actual noise environment. The filtering criteria relate mainly to: (i) the presence of a microphone bias (via manual or automatic calibration), and (ii) the data location accuracy so as to ensure that the actual position of the user has a probability of $68 \%$ to be within a 15 meters radius circle around the given location. This filtering resulted in keeping only 198,197 measurements (less than $10 \%$ collected) out of the initial dataset, to aggregate with the other types of noise data. This points out the rather low reliability, and hence usability, of MPS noise measurements if gathered passively in the background.

It is interesting to further analyze the "quality" of the crowdsensed data in the (default) passive background versus proactive journey mode, provided in the two rightmost columns of Table 1. We see that while the Journey mode contributes with quantitatively less data that the background mode (23,469 out of the 2.4 million measurements under scrutiny), the ratio of qualitatively relevant data is significantly higher with the Journey mode (11,669 data points that is about $50 \%$ of the overall data points in the Journey mode versus less than $1 \%$ for the automatic measurements). This observation highlights the significance of both the technical (continuous, 5 seconds long 
Table 1. Filtering the Ambiciti data crowdsensed in Paris by 949 users over the year 2017.

\begin{tabular}{llccc}
\hline & Condition & All Data & Journey Mode & Automatic Mode \\
\hline Samples & & $2,434,018$ & 23,469 & $2,410,549$ \\
Location accuracy & $<15 m$ & 198,197 & 11,669 & 186,528 \\
bias & $\neq 0$ & $1,193,052$ & 15,081 & $1,177,971$ \\
Still & Accuracy $>95 \%$ & 792,601 & 3,209 & 789,392 \\
In vehicle (bus, metro) & Accuracy $>95 \%$ & 8,886 & 9 & 8,877 \\
Walking & Accuracy $>95 \%$ & 26,529 & 2,979 & 23,550 \\
\hline
\end{tabular}

measurements) and behavioral (manually activated, dedicated to specific paths, and making the user active) conditions of measurements.

\subsection{MPS increases the environmental consciousness and the sensitiveness to the noise exposure}

The urban-scale experiments that we ran not only allowed us to assess the contributions (and related conditions) of MPS-based measurements to noise mapping, but they also enabled us to study the motivations and practices of Ambiciti users. We specifically published a supporting online questionnaire from June to December 2017, which included 21 multiple-choice and 6 open-ended questions ${ }^{4}$; it was structured in three parts: (i) the technical and practical contexts of use of the Ambiciti app, (ii) the user's motivations for downloading and using the app, and (iii) the user's perception after using it. Table 2 provides various characteristics of the Ambiciti users who answered the questionnaire.

We gathered 66 contributions to the questionnaire, out of which the following motivations arose: assessing the quality of life in relation to the individual exposure to noise (50\% of the respondents); sensitivity to a better knowledge of the effects of the environmental pollution on own health (30\%); access to relevant information toward better health care and initiative for better protection against pollution (30\%); and ability to confront the perceived noise levels against the measured ones, notably in green areas or close to roads (25\%). This feedback illustrates the capacity of an MPS app to make citizens more conscious of the urban noise and to contribute to changing their perception of their neighborhood. Although about $75 \%$ of the surveyed users declared that using Ambiciti does not influence their itineraries, 20\% believed they unconsciously choose less polluted routes thanks to the provided information and $5 \%$ declared having actually changed their usual itinerary.

MPS-based noise monitoring is mainly perceived as giving evidence of environmental pollution. For the contributing individuals, the measured noise level is "evidence" confirming what was previously just a feeling that a place is noisy. Nevertheless, the demonstrative function of the crowdsensed data remains poorly legitimated by the decision-makers. This is due to the relatively low accuracy of the smartphone microphones as noise sensors, but also to the assumed non-expertise of the users. We may consider, though, that the measurements of noise levels using a MPS app provide relevant information to sensitize citizens and decision-makers about the individual and collective exposures to noise and their impact on health, if the the appropriate MPS protocol is implemented.

${ }^{4}$ https ://docs .google.com/forms/d/1LAQN3qCfe7EOFdzzCRPkKznRxUgJB9SeyhNfMmJDDSs 
Table 2. Characteristics of the Ambiciti users who answered the questionnaire

\begin{tabular}{llc}
\hline Feature & Class & Percentage \\
\hline \multirow{2}{*}{ Gender } & Male & 59 \\
& Female & 41 \\
\hline \multirow{2}{*}{ Age } & $<25 y$ & 10 \\
& between 25y and 50y & 65 \\
\multirow{2}{*}{ Context of Use } & $>50 y$ & 25 \\
& Private & 63 \\
& Professional & 37 \\
\hline \multirow{2}{*}{ Sector (Work) } & Environment & 34 \\
& Public Policy, Urban planning & 28 \\
& IT & 22 \\
& Others & 16 \\
\hline
\end{tabular}

\subsection{Toward informing noise policies}

The experiments that we ran in Paris show that, subject to the adequate filtering of the collected data, MPS allows capturing non-predictable and various noise events (and other pollutants cross effects (Fecht et al., 2016)). A more representative knowledge of the actual urban soundscapes is the first step to better understand the relationships between noise, health, and well-being. The contribution of urban dwellers to MPS remains to be strengthened and improved. Indeed, the effectiveness of MPS requires overcoming the poor default accuracy of the contributed measurements. Reaching a critical mass of users, and of contributed data across space and time, is a condition for increasing the representativeness of noise maps optimized using crowdsensed data. Further, decision-makers could engage more pro-actively in strategies for reducing noise exposure, including the use of mobile crowdsensing to inform the knowledge about such individual and collective exposure. Beyond the specific case of Paris, some local decision-makers we interviewed have declared that considering a better knowledge of the actual urban noise environment conflicts with more global territorial strategies that aim at building an attractive image of their city and the living conditions it offers. This socio-economical paradox raises a fundamental issue. Ideologically and organi-

485 zationally, institutions have to strike a balance between, on the one hand, providing reliable information about the noise environment -and its possible adverse effects on health or well-being- and, on the other hand, building a positive image of their city for its effective, sustainable development.

\section{MPS to better observe the related effects of noise on the health and well-being}

Even though more accurate representations of the urban noise across time and space are possible using available technologies -including MPS-, a gap remains to correlate them with health issues and well-being. Indeed, many individual and collective parameters influence the perception and impact of noise (e.g., physical and mental activity, state of mind and irritability, social context). The ability to infer a meaningful relationship between the exposure to noise and health issues thus requires: (1) the long-term combined observation of the environmental noise and the health symptoms of the exposed population, and (2) focusing on a specific population in a specific spatial context so as to minimize (or take into account) the influence of other parameters (than noise) on the evolution of the physiological and social characteristics of this population. 


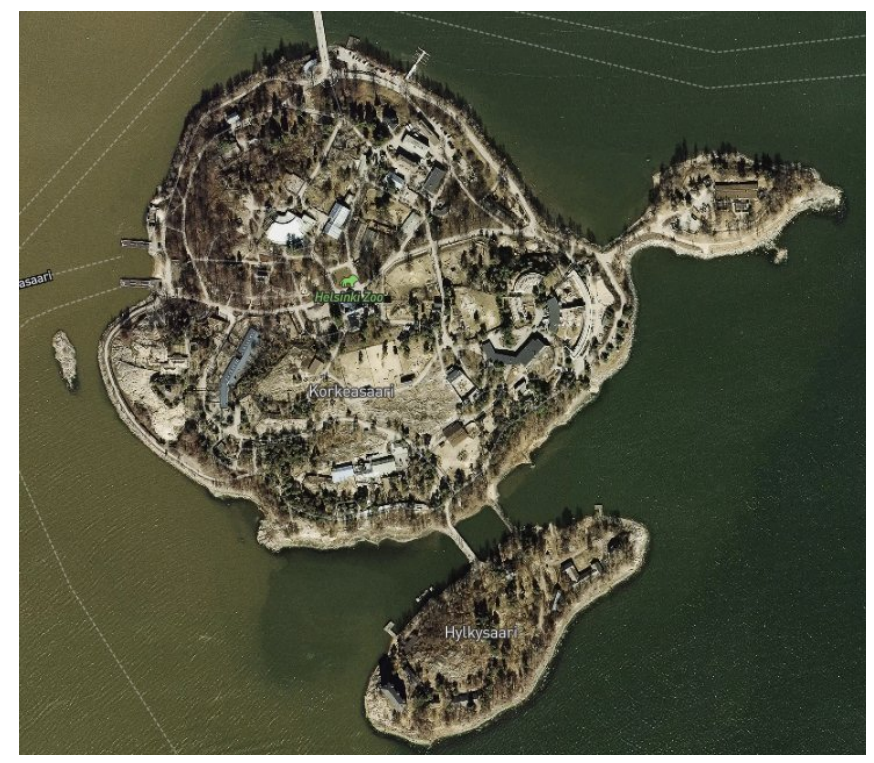

Figure 7. Satellite view of the Korkeasaari island, Helsinki, Finland.

\subsection{The challenge of isolating the impact of noise on health}

Some studies in schools (Goswami, Hassan, \& Sarma, 2018; B. Shield \& Dockrell, 2008) or occupational environments (Concha-Barrientos et al., 2004) observe relationships between noise levels and stress or tired feelings. But, even if these studies consider specific environments that are relatively "enclosed", their authors often point out the difficulty in establishing causal relationships.

We then sought to monitor noise and health symptoms together, in a context that is sufficiently enclosed to be able to ignore other environmental or behavioral parameters that would also affect the being's welfare. An opportunity arose through a collaboration with the Municipal Zoo and the Innovation Unit (Forum Virium) of the City of Helsinki, Finland. The whole Korkeasaari island is dedicated to the zoo and the keeping of the animals. The protection regulations about the animals' welfare require that the captive livestock should not be exposed for a long time period to noisy environments. Indeed, such exposure increases the risks of disease and social behavior disruptions. For their organizations and interactions, animals are much more sensitive to the combination of noise and vibrations than human beings (Basner et al., 2015). Many biological studies observed the impact of noise on wild animals (Nabi et al., 2018; Raap, Pinxten, Casasole, Dehnhard, \& Eens, 2017; Rosa \& Koper, 2018; Troïanowski, Mondy, Dumet, Arcanjo, \& Lengagne, 2017), but only a few report long time observations of several species in common urban environments (Shannon et al., 2015).

In Helsinki, the Korkeasaari Island (See Figure 7) is situated about 300 meters from the seafront where construction work is scheduled from 2017 to 2025 . The construction of large residential buildings (capacity of 20,000 new inhabitants) and related urban planning projects, including a tramway lane on a new dedicated bridge, are impacting the noise environment directly near the zoo area and will do so over the eight year period. Preliminary studies conducted by the local biologists show that some species, like tigers, bearded vulture, and markhors, are most impacted by noise, notably due to the situation of their living place. But this changing and unpredictable noise envi- 
ronment could affect animals more globally. Thus, the biologists must enhance their capacity to identify excessive noise exposures, to ensure the welfare of the most sensitive animals. This can be done with specific noise-protection installations or moving animals temporarily to other parts of the zoo.

Although the observed population is not human, the specific conditions of these captive living beings provide a spatially and temporally stable research environment, notably a fixed situation, routine behaviors, and recurrent environments. Further, as highlighted above, the ongoing construction work nearby the Helsinki zoo calls for the monitoring of the environmental noise in relation to the behavior and health of the animals. Inria, Forum Virium and the biologists of the zoo have thus conceived an original methodology to correlate the variations of the noise environment and the animals' health (notably stress) and behavior. The experiment is specifically focused on closely monitoring the evolution of the environmental noise using MPS, together with studying its impact on the animals, in particular through biological analyses that will monitor the evolution of the hormones involved in stress phenomena.

\subsection{MPS protocol to evaluate the effects of noise: Experiment with monitoring the health and social behavior of animals at the Helsinki $z o o$}

Noise measurements using the Ambiciti app will be carried out in the zoo over the 550 duration of the construction work, that is, from the end of 2017 till 2025-2026. Technically, nine calibrated mobile phones running the Ambiciti app will monitor the noise levels. Six of the nine devices further use external rather than inbuilt microphones to enhance the measurements accuracy (Kardous \& Shaw, 2016). The on-going experiment is conceived around two main steps.

Step 1: A specific protocol of measurements. Over the eight years of the experiment, the employees of the zoo will collect noise measurements in the winter (when the zoo is closed) and summer (the peak season), as well as during special occasions (school activity days, big events especially during late nights). Under the direction of Dr. Kirsi Pynnönen-Oudman, Animal Physiologist, the team has already conducted two sets of measurements: in January 2018 for two weeks (the zoo was closed to the public during this period) and in July 2018 (with visitors). They placed three mobile phones at strategically located sites on the island. The employees of the zoo used the six remaining mobile phones (with external mics) to make continuous, 5 second long measurements during their work time. The external microphones not only provide better accuracy but also make it possible to keep the phone in a ventral pocket while leaving the microphone unobstructed. The bias of each microphone was evaluated via a calibration process by reference to a professional sound level meter.

63,025 observations have been collected during the January session and 66,512 in July. Out of them, 54,947 and 60,493 observations, respectively, have relevant enough location accuracy, under $25 \mathrm{~m}$ (see Figure 8 ). These observations have been interpolated via the kriging method (Aumond et al., 2018) ${ }^{5}$ across space and time. This probabilist technique ensures a relevant enough representation of the whole area on the basis of specific locations and moments of measurements. The collection of the mobilesensed data allowed the production of hourly noise maps for these two sessions of 575 measurements (see Figure 9), which provide relevant and dynamic information about

\footnotetext{
${ }^{5}$ See also: http://www.gisresources.com/types-of-interpolation-methods_2/, accessed 03/19.
} 


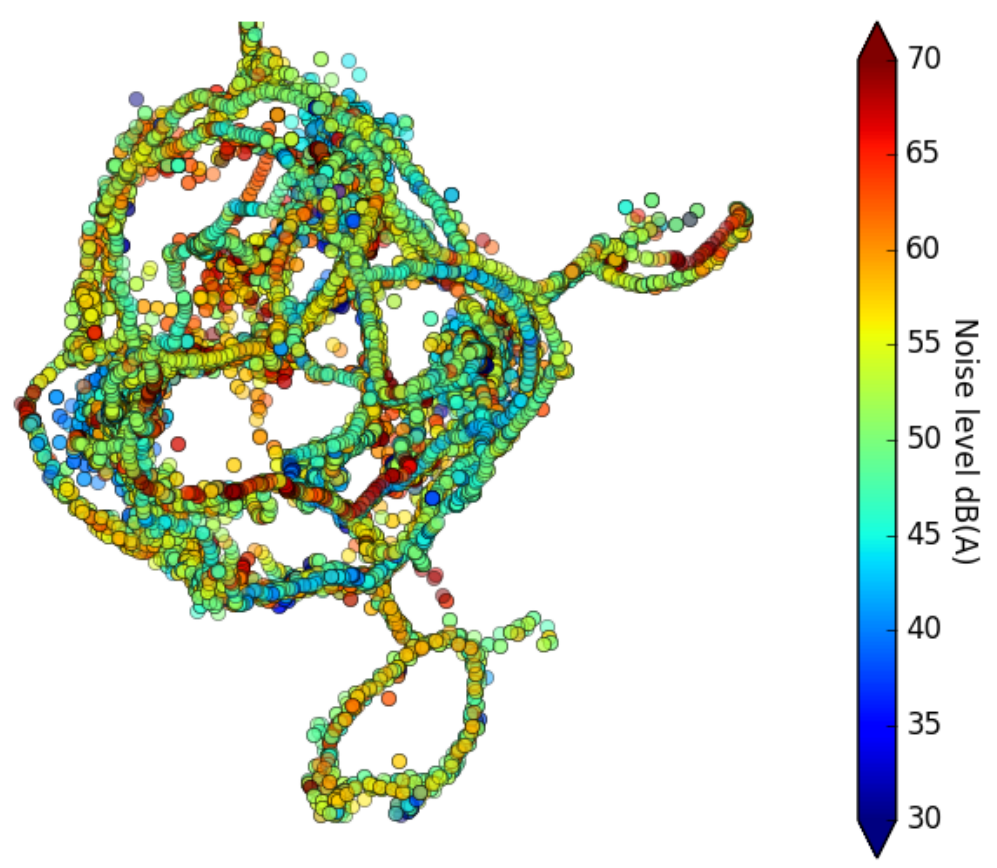

Figure 8. The collection of crowd-sensed noise data from the January 2018 measurements session at the Helsinki zoo.

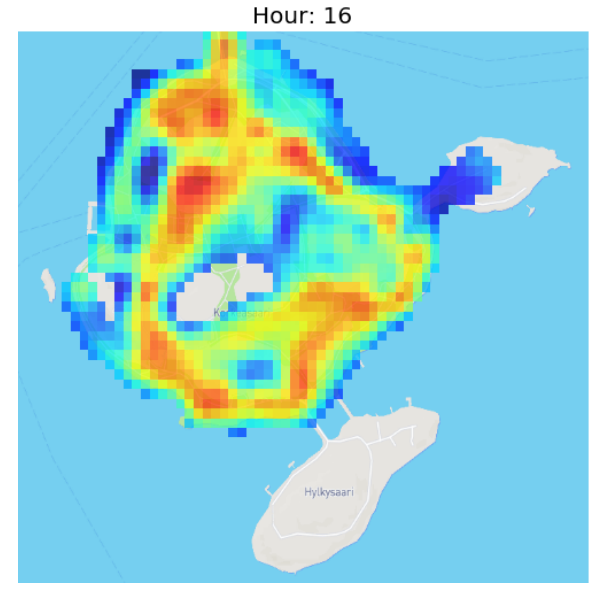

(a) January 2018
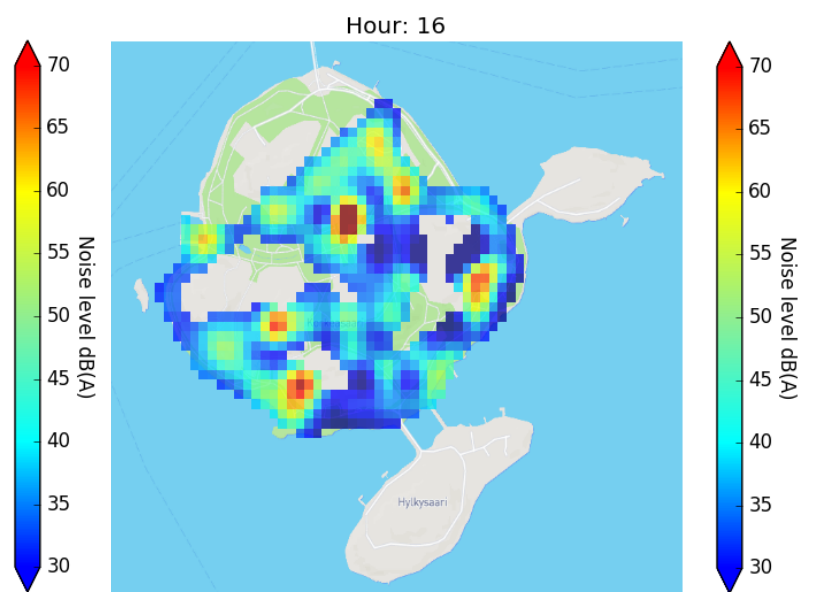

(b) July 2018

Figure 9. Noise maps of the Helsinki zoo computed from crowdsensed measurements. Average levels by session, at $4 \mathrm{pm}$.

the noise environment in the zoo area.

Step 2: Correlating noise and animals behavior and physiology. The successive periods of measurement will provide a referential noise environment, which takes into account the noise impacts of the recurrent phenomenon like seasons or visitors activity. These referential resources will make it possible to evaluate the future noise effects of the construction works on animals' well-being. From the same time, the biologists will observe the animals' behaviors and potential evolution of their metabolisms. Notably, from 2019, they will measure the levels of cortisol hormone in the animals' se- 
cretions to evaluate the possible stress effects of excessive noise. Many studies observed the increase of cortisol concentrations in response to stressors like increasing noise and to regulate metabolic rates as blood glucose (Ash, Smith, Knight, \& Buchanan-Smith, 2018; Jimeno, Hau, \& Verhulst, 2018; Kleist, Guralnick, Cruz, Lowry, \& Francis, 2018; Willen, Mutwill, MacDonald, Schiml, \& Hennessy, 2017). The physiologist of the zoo has defined non-invasive methods, that is, no blood sampling but rather regular behavioral observations and fecal sampling. The animals (bearded vultures, markhors, tigers, lions, amur leopards) that are close to the construction area will be the initial target since cortisol measurement at a large scale is expensive.

\subsection{Toward informing the health and social effects of noise}

The noise monitoring experiment at the Helsinki zoo focuses on animals enclosed in constricted places and with routine behaviors. Dr. Pynnönen-Oudman is aware of the difficulty of differentiating the effects of the construction noise and vibrations with the effects of internal noise (public, machines) on the animals. Disposing of a referential noise map of the soundscape before the beginning of the construction should help. The physiological and behavioral monitoring of the animals will provide information about the accumulated effects of the environmental noise they are exposed to. Still, beyond providing original knowledge about the animals' reaction to noise in the specific context of an urban zoo, such an experiment can also be considered a first step toward pointing out the direct effects of environmental noise on the health (cortisol monitoring), wellbeing and social behavior (observation of individual attitudes, social interactions and reproduction activities) of living forms.

The City of Helsinki is developing a multi-faceted strategy for promoting the local environmental quality of life. Since 2014, a noise prevention action plan aims at reducing traffic noise, protecting sensitive sites like the zoo, and preventing the spreading of noise, notably around the harbor area ${ }^{6}$. This environmental public policy is combined with operational strategies designed by the Innovation department Forum Virium. This partnership makes possible the undertaking of pro-active programs for reducing exposure to noise, which are at the same time consistent with territorial sustainability and relevant to a very specific local problem.

\section{MPS to evaluate the subjective perception of environmental noise}

${ }_{615}$ A better understanding of the effects of noise on health and well-being must take into account the following duality: when exposed to noise, humans are physiologically affected by an acoustic vibration, and, at the same time, mentally informed by significants that they interpret to subjectively operate better in their environment. Independent of the individual sensitivity to noise, the relation between the physical 620 noise environment (i.e., the noise levels measured in $\mathrm{dB}(\mathrm{A})$ ) and its perception by a person is a complex function due to the impact of the person's context on the latter. For instance, a person's habit or focus on a complex task is "attenuating the disruptive effects of noise" (Basner et al., 2015). Hence, we need to be able to monitor the physical characteristics of the noise environment together with its perception by humans in their social life practices (Chung, To, Lai, \& Choy, 2014), the individuals' sensitiv-

\footnotetext{
${ }^{6}$ https://www.portofhelsinki.fi/en/port-helsinki/environmental-responsibility/management -environmental-impacts, accessed 03/19.
} 


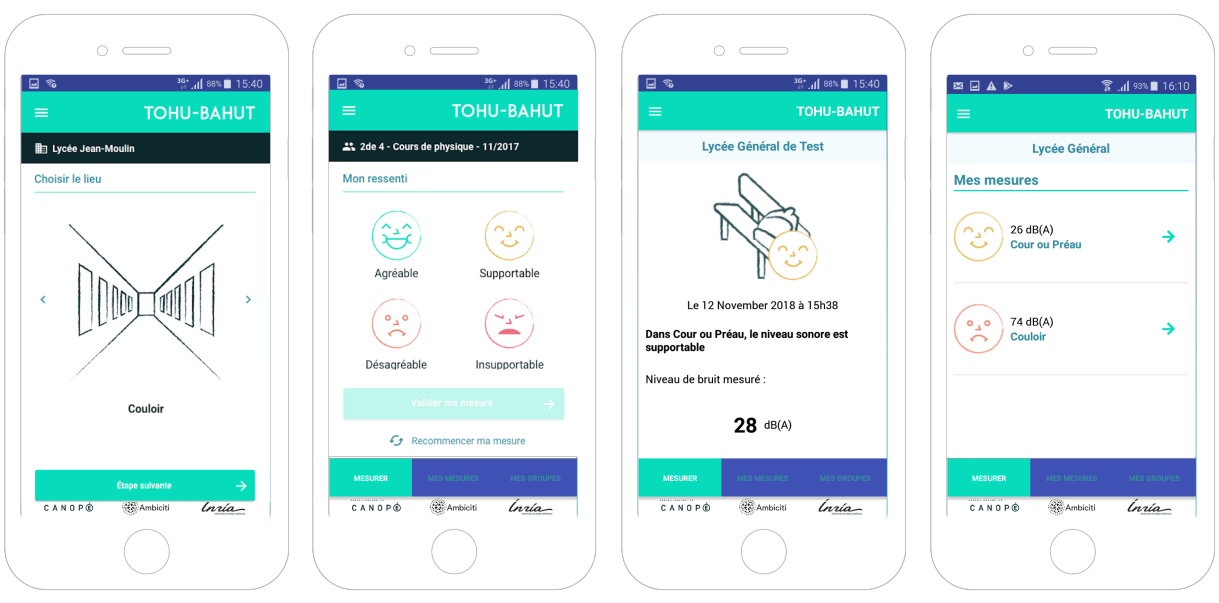

Figure 10. Screenshots of the interface of the Tohu-Bahut app. (a) The user can scroll to select the area, then $(b)$ a feeling of the soundscape, while the noise level is measured. (c) The result of the localized noise measurement and its perception by the user is displayed. (d) Stats are provided from the average noise levels and feelings for each area.

ities and the contextual cognitive situations. Through the gathering of individualized information, MPS has the potential to contribute such knowledge.

\subsection{Protocol to evaluate the subjective perception of noise by individuals}

To assess the relevance of MPS to gather jointly the physical and subjective percepof the environmental noise, we have been studying the related customization of the Ambiciti app. We specifically undertook a collaboration with the Canopé French national center for digital teaching resources to develop the Tohu Bahut app for the school environment. Tohu Bahut enables the students and teachers to individually report their subjective feeling about the environmental noise they are exposed to while the noise level is quantitatively measured in $\mathrm{dB}(\mathrm{A})$ in the background. The app further distinguishes the representative school areas (classroom, study room, hall, cafeteria, schoolyard, outside the school) for the reporting.

The Tohu Bahut user interface is as follows (see Figure 10): (1) the user must first state the school area where he/she is standing using the given predefined icons; (2) the user then qualifies the perceived sound environment using four predefined smiley icons that respectively indicate a Pleasant, Tolerable, Intolerable or Irritating feeling; at the same time and in the background, the app measures the noise levels in $\mathrm{dB}(\mathrm{A})$ for 15 seconds; and (3) finally, the app displays the measured level next to the expressed feeling.

\subsection{Assessing the contribution of MPS to collect the users' perceptions: Evaluating soundscapes in schools}

For years, the reduction of the speech intelligibility has been considered a major consequence of the students' exposure to noise (B. Shield \& Dockrell, 2008), as it affects the students' performance and well-being. Recent studies show that students (and teachers) are exposed to noise levels that are much higher than the commonly accepted recommendations (Goswami et al., 2018). The ideal ambient noise levels, defined as 


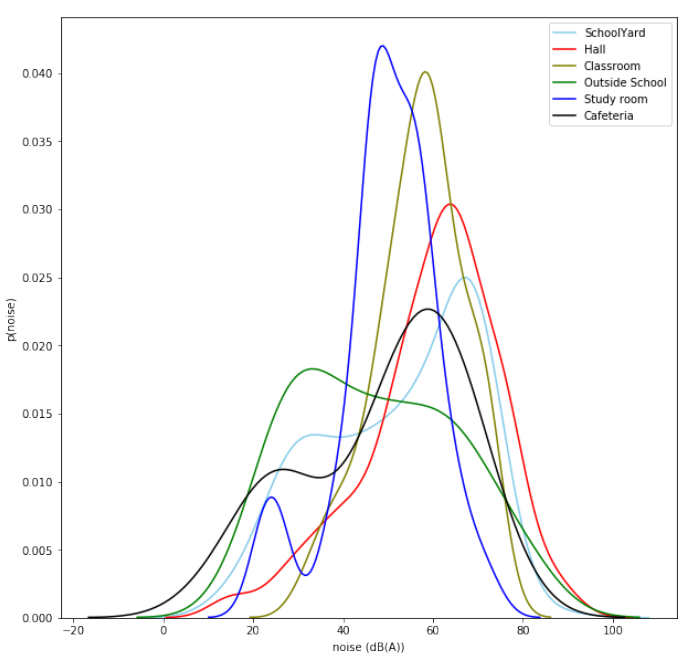

(a) for each place.

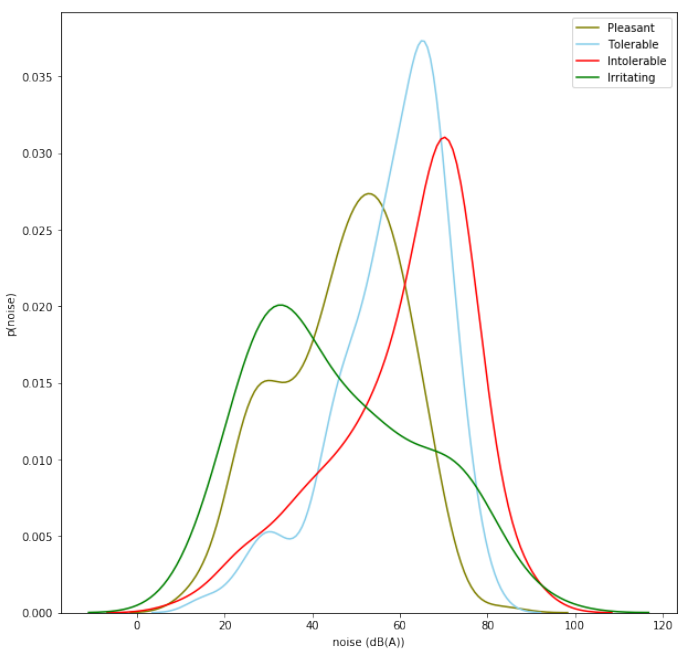

(b) for each feeling.

Figure 11. Distribution of noise samples regarding (a) the type of place in schools, (b) the perceived feelings of the noise environment.

allowing clear speech recognition, are fixed at $35 \mathrm{~dB}(\mathrm{~A})$ for empty classrooms and 56 dB(A) in occupied classrooms (Brill, 2017; B. M. Shield \& Dockrell, 2003).

The measured noise levels. As first experiments using the Tohu Bahut app, we collected 646 observations from 73 students from 28 different middle schools, over a 7 month period (from January to July 2018). The measurements were gathered during school time under the supervision of a teacher in Technical, Biological or Environmental Science classes. With a pedagogical objective, the students collectively sensed noise using the app. Out of the collected observations, $36.7 \%$ refer to noise levels under 50 $\mathrm{dB}(\mathrm{A}), 48.8 \%$ between 50 and $70 \mathrm{~dB}(\mathrm{~A}), 13.8 \%$ between 70 and $85 \mathrm{~dB}(\mathrm{~A})$ and only 5 observations measured levels exceeding $85 \mathrm{~dB}(\mathrm{~A})$. Most of the measured noise levels were in the range of 30 to $80 \mathrm{~dB}(\mathrm{~A})$, with significant peaks between 50 and $70 \mathrm{~dB}(\mathrm{~A})$. Figure 11(a) shows, as expected, that the Study rooms (levels from 20 to $70 \mathrm{~dB}(\mathrm{~A})$ ) and the Classrooms (from 30 to $80 \mathrm{~dB}(\mathrm{~A})$ ) are globally the quietest places. However, most of the measured levels in Classrooms are between 50 and $75 \mathrm{~dB}(\mathrm{~A})$ high, partly significantly over the $56 \mathrm{~dB}(\mathrm{~A})$ recommended level for occupied classrooms. The Hall and the Schoolyard are the noisiest (respectively, 45 to $95 \mathrm{~dB}(\mathrm{~A})$, and 20 to $90 \mathrm{~dB}(\mathrm{~A})$ ); significant peaks are highlighted at about $70 \mathrm{~dB}(\mathrm{~A})$ for these places. Although we do not know the duration of exposures, the higher levels in the Hall and the Schoolyard are in the critical range for humans' health. The Cafeteria can be very noisy, with measured levels over $90 \mathrm{~dB}(\mathrm{~A})$. Finally, a wide range of noise levels can be observed outside the school and in the cafeteria, with levels between $20 \mathrm{~dB}(\mathrm{~A})$ and more than $100 \mathrm{~dB}(\mathrm{~A})$. These figures show a high concordance between the expected and actual noise levels in the various school areas. More importantly, they exhibit a high prob675 ability that students will be exposed to noise levels over $70 \mathrm{~dB}(\mathrm{~A})$ and up to 100 $\mathrm{dB}(\mathrm{A})$.

The noise level and its perception by individuals. The study of the perception of the noise environment shows significant deviation from the a priori expectation 


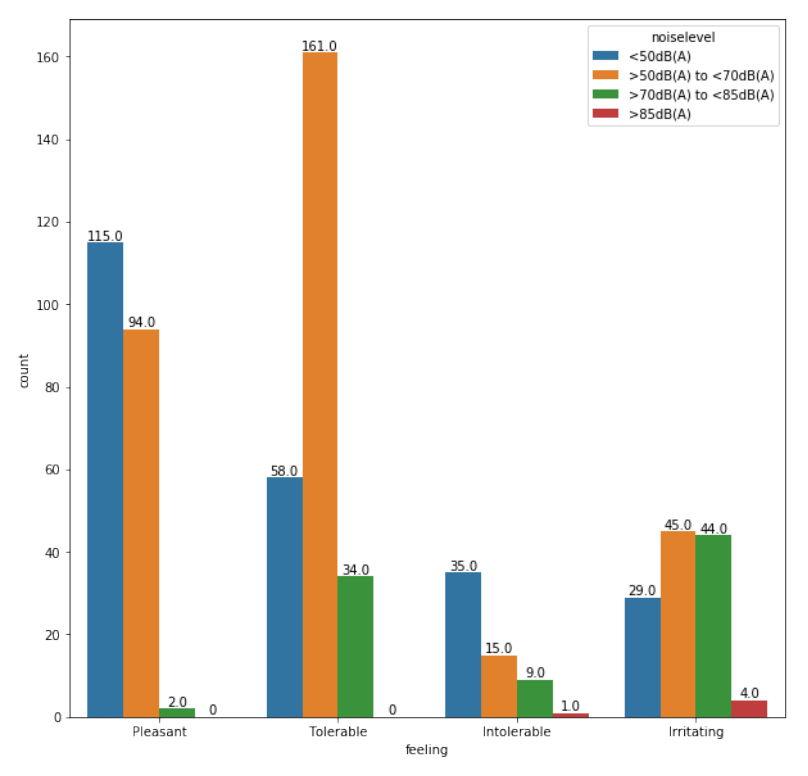

Figure 12. Distribution of the noise levels per perceived feeling of the noise environment.

(see Figure 11(b)). We observe that "Pleasant" environments refer to measurements between 20 to $75 \mathrm{~dB}(\mathrm{~A})$, with significant peaks at $30 \mathrm{~dB}(\mathrm{~A})$ and $55 / 60 \mathrm{~dB}(\mathrm{~A})$, while such a qualifier would have been expected for levels under $50 \mathrm{~dB}(\mathrm{~A})$. The "Tolerable" environments (a priori expected in the [50..70] $\mathrm{dB}(\mathrm{A})$ range) are in the range of 40 to $85 \mathrm{~dB}(\mathrm{~A})$, with a very high peak at $65 / 70 \mathrm{~dB}(\mathrm{~A})$. "Intolerable" feelings concern 20 to $90 \mathrm{~dB}(\mathrm{~A})$ ambiances, with a peak at $70 / 80 \mathrm{~dB}(\mathrm{~A})$. Lastly, "Irritating" perceptions concern a vast spectrum of noise levels, from 10 to $85 \mathrm{~dB}(\mathrm{~A})$, with a peak at $30 \mathrm{~dB}(\mathrm{~A})$. Quiet environments (between 40 and $60 \mathrm{~dB}(\mathrm{~A})$ ) are thus perceived as "Pleasant" or "Tolerable", but for a non-negligible part as "Irritating". In contrast, a significant part of the 50 to $80 \mathrm{~dB}(\mathrm{~A})$ environments are perceived as "Pleasant" or "Tolerable".

Figure 12 shows the distribution of the stated perceptions with respect to the ranges of measured noise levels. We observe that about half of the "Pleasant" perceptions relate to noise levels under $50 \mathrm{~dB}(\mathrm{~A})$ (115 selections out of 211, 55\%); $99 \%$ (209 on 211) relate to levels under $70 \mathrm{~dB}(\mathrm{~A})$ (level for a usual conversational speech). This result illustrates a very strong consistency between the perceived and actual noise level. A similar observation concerns the "Tolerable" perceived environments: $64 \%$ correspond to noise levels between 50 and $70 \mathrm{~dB}(\mathrm{~A})$, and $87 \%$ under $70 \mathrm{~dB}(\mathrm{~A})$. In contrast, the environments perceived as "Intolerable" refer at $83 \%$ to noise levels under $70 \mathrm{~dB}(\mathrm{~A})$ (50 out of 60 measurements) and even $35 \%$ to levels under $50 \mathrm{~dB}(\mathrm{~A})$. Similar observations concern the "Irritating" perceived environments, with $61 \%$ (74 out of 122 measurements) related to noise levels under $70 \mathrm{~dB}(\mathrm{~A})$ and $24 \%$ under $50 \mathrm{~dB}(\mathrm{~A})$. These results suggest a stronger correlation between perceived and measured ambient noise in actual "quiet" environments than in "noisy" environments. Quiet environments under $70 \mathrm{~dB}(\mathrm{~A})$ have more probability to be perceived as "Intolerable" or "Irritating" (90\% data) than noisy environments (more than $70 \mathrm{~dB}(\mathrm{~A})$ ) to be perceived as "Pleasant" or "Tolerable" ( $1.5 \%$ of the data).

Overall, the observations gathered in the schools illustrate that the actual physical noise level does not straightforwardly map to how the user feels about the soundscape. The same level of sound (i.e., $65 \mathrm{~dB}(\mathrm{~A})$ ) can be perceived as "Pleasant" for some 
and "Irritating" for others. Still, this shows the influence of the context, which, in our experiment, is primarily the specific school area. The area (classroom, hall, etc.) and the state of mind that is associated with it (more or less sensitive to noise) likely influence the perception of the noise by the young middle-school students. We stress that the documented results must be considered as experimental due to the -so far- relatively small amount of collected data and to the -although supervised- selfreporting by the students. However, our study emphasizes the relevance of MPS to better understand the subjective sensitivity to environmental noise. Such an approach may further be complemented with the evaluations of stress and tired feeling levels so as to ultimately inform the impact of the soundscapes on the people's health and well-being. The study in the school environment is moreover a valuable approach to also demonstrate the impact of soundscapes on learning capacities.

Further studies for better understanding the variation between actual and perceived levels of noise. The MPS of soundscapes provides the possibility to measure an actual level of environmental noise and for a user to declare its subjective perception. Correlating these two parameters can thus help us in better understanding the effects of noise on individuals' health and well-being. Notably, we observed a variation between the measured and perceived levels of noise, significantly more pronounced in noisy environments. Further experiments could be easily carried out in other social contexts than schools and could include physiological monitoring.

\section{Conclusion}

To address citizens' demands and institutions' incentives (EU, WHO), decision-makers

\section{have to deal with the dual status of the urban noise environnent. On the one hand, and} depending on individual sensitivities, the exposure to noise is the cause of major health issues. On the other hand, soundscapes play a social role and, in specific contexts, urban noise contributes to the citizens' well-being. Whatever the strategies to balance the negative and positive effects of the urban environmental noise, the physical and subjective perception of the urban noise remain poorly evaluated and need to be better known, from both individual and epidemiological standpoints.

To overcome the above, this paper has advocated that Mobile Phone Sensing (MPS) is a promising complement to the state-of-the-art approaches to urban noise monitoring that leverage mathematical modelling and professional sensors. We have reported three supporting MPS experiments, for which we designed specific protocols, tools and methodologies. These protocols focus on different objectives, leading to studies in specific social and spatial contexts.

The urban-scale experiments (Section 3), especially the ones carried out in Paris in 2016, have shown that MPS can contribute to make visible soundscapes related to human activity at a high spatial resolution. Thanks to specific modes of measurement that involve the users pro-actively, the relevance of the collected data can significantly increase and balance the a priori, relatively low accuracy of the data sensed by phones' microphones. With the help of algorithmic error correction, data assimilation, and interpolation, the sensed data can contribute to a better knowledge of the actual noise environment with high spatio-temporal resolutions. This representative knowledge is a first step for correlating environmental noise and health issues or well-being. A critical mass of contributors and a greater involvement of policy-makers remain obstacles to overcome for more relevant representations of the urban noise environment, at a larger 
scale.

Even if concerning animals, the on-going MPS experiment at the zoo of Helsinki (Section 4) aims at providing original resources to better relate the direct effects of a changing noise environment with behavioral and physiological disruptions. Thanks to the implementation of a tailored protocol in such a specific environment with the engagement of the contributing users -the zoo personal-, MPS enables a long-term monitoring strategy at low cost, both economically and regarding the involved social practices. Obviously, the animals' sensitivities to sound, physiological responses and social behaviors differ from the ones of humans. Nevertheless, the results of the MPS experiment at the Helsinki zoo will likely bring new methodological insights to study the impact of noise on health and well-being disruptions.

765 The third and last experiment that we reported (Section 5) shows that MPS can contribute to a better understanding of the subjective perception of soundscapes with respect to specific activities and places. The MPS app enables combining subjective individual feedback from users' perception of noise and collective analysis, which could help epidemiological work. Further work should correlate the actual and perceived noise environments with well-being, concentration or tired feelings of students.

Although the presented experiments highlight the added-value of Mobile Phone Sensing to a better knowledge about the urban noise and soundscapes, we must take into consideration its technological, cultural and social limits. The currently limited accuracy of the embedded sensors induces a still relatively poor relevance of the sensed ta. We saw that the correction of the mobile bias and appropriate conditions of measurement could improve this relevance. Our research also points out the relevance of collective sessions of noise sensing, which provide significantly more accurate data and contextual information about the conditions of their production, notably thanks to a more pro-active involvement of the users in the sensing process.

780 Beyond their strategic and methodological specifics, the reported experiments highlight the relevance of participatory noise sensing in contributing to increased knowledge not only of the urban noise environment but also of its subjective perception. More globally, subject to a critical mass of contributed measurements, MPS can increase the accuracy of noise maps and forecasts at the scale of the city (and potentially worldwide). MPS as a -both quantitative and qualitative- resource for the monitoring of the urban noise and soundscapes still needs to be enhanced and integrated with epidemiological health or well-being studies. Lastly, and although there already are many ongoing programs, more inter-disciplinary studies, and contextualized methodologies including MPS technologies and protocols should increase the sensitization of citizens, professionals and decision makers for reducing the urban environmental noise, its effects on health, and for making cities more attractive and sustainable for any living form.

Acknowledgements: The authors would like to thank: Ambiciti for granting us access to the specific data as part of research collaboration; the City of Helsinki, notably

795 Forum Virium; and Professor Kirsi Pynnönen-Oudman who is managing the experiment for the zoo. The authors also acknowledge the support of the CityLab@Inria research program on digital solutions contributing to socially and environmentally sustainable cities. 


\section{References}

Deloite, C. (2017), Global mobile consume

www2.deloitte.com/content/dam/Deloitte/us/Documents/technology-media -telecommunications/us-global-mobile-consumer-survey-second-edition.pdf

840

Dorst, K. (2015). Frame creation and design in the
Design, Economics, and Innovation, 1(1), 22-33.

Fecht, D., Hansell, A. L., Morley, D., Dajnak, D., Vienneau, D., Beevers, S., ... Gulliver, J. (2016). Spatial and temporal associations of road traffic noise and air pollution in london: Implications for epidemiological studies. Environment International, 88, 235-242.

Fu, W., Wang, C., Zou, L., Liu, Q., Gan, Y., Yan, S., ... Cao, S. (2017). Association between exposure to noise and risk of hypertension: a meta-analysis of observational epidemiological studies. Journal of Hypertension, 35(12), 2358-2366.

Genuit, K., \& Fiebig, A. (2006, November). Psychoacoustics and its benefit for the soundscape approach. Acta Acustica united with Acustica, 92, 952-958.

gostinelli, S. (2009). Comment penser la médiation inscrite dans les outils et leurs dispositifs? Une approche par le système artefactuel. Distances et savoirs, 7(3), 355-376.

artphone user accessed close to 40 app Retrieved 10/03/2019, from https://www.appannie.com/en/insights/market

ical stress in the common marmoset (callithrix jacchus): Validation of a salivary cortisol collection and assay technique. Physiology 83 Behavior, 185, 14-22.

C. (2017). Acoustic mapping based on measurements: space and time interpolation. INTERNOISE and NOISE-CON Congress and Conference Proceedings, 255(5), 2287-2298. C. (2018). Kriging-based spatial interpolation from measurements for sound level mapping in urban areas. The Journal of the Acoustical Society of America, 143(5), 2847-2857.

Basner, M., Brink, M., Bristow, A., de Kluizenaar, Y., Finegold, L., Hong, J., .. Sörqvist, P. (2015). ICBEN review of research on the biological effects of noise 2011-2014. Noise and alth, 17(75), 57-82. pen, E. V. (2017). Noise in Europe 2017: Updated assessment (Tech. Rep.). Bilthoven, Netherlands: European Topic Centre on Air Pollution and Climate Change Mitigation (ETC/ACM).

Bonfils, P. (2015). Immersion et environnements numériques: une approche mthodologique $K$-12 classrooms (Unpublished master's thesis). Faculty of The Graduate College at the University of Nebraska.

, J (2015). Roles of smartphone app use in improving social capital and reducing socia of inhabitants of cities. In 11th International Congress on Noise as a Public Health Problem (ICBEN) (pp. 1-8). Nara, Japan.

Concha-Barrientos, M., Nelson, D. I., Driscoll, T., Steenland, N. K., Punnett, L., Fingerhut, M., ... Corvalan, C. (2004). Selected occupational risk factors. In Comparative quantification of health risks (Vol. 2, pp. 1651-1801).

Gille, L.-A., Marquis-Favre, C., \& Weber, R. (2017). Aircraft noise annoyance modeling: Con-

sideration of noise sensitivity and of different annoying acoustical characteristics. Applied 
Acoustics, 115, 139-149.

Goncalves, J., Hosio, S., Rogstadius, J., Karapanos, E., \& Kostakos, V. (2015). Motivating participation and improving quality of contribution in ubiquitous crowdsourcing. Computer networks, 90, 34-48.

Goswami, B., Hassan, Y., \& Sarma, A. (2018, January). The effects of noise on students at school: A review. International Journal of Latest Engineering and Management Research (IJLEMR), 03(01), 43-45.

Hachem, S., Mallet, V., Ventura, R., Raverdy, P. G., Pathak, A., Issarny, V., \& Bhatia, R. (2015, March). Monitoring Noise Pollution Using The Urban Civics Middleware. In Proceedings of IEEE First International Conference on Big Data Computing Service and Applications (pp. 52-61). Redwood City, CA.

Halim, H., Abdullah, R., Mohd. Nor, M. J., Aziz, H. A., \& Rahman, N. A. (2017). Comparison between measured traffic noise in Klang Valley, Malaysia and existing prediction models.

865 Engineering Heritage Journal (GWK), 1, 10-14.

Issarny, V., Mallet, V., Nguyen, K., Raverdy, P. G., Rebhi, F., \& Ventura, R. (2016, December). Do's and Don'ts in Mobile Phone Sensing Middleware: Learning from a Large-Scale Experiment. In Proceedings of the 17th international middleware conference (pp. 17:1-17:13). Trento, Italy.

Jambrošić, K. (2017, December). Introduction to psychoacoustics and psychoacoustic tests. In DENORMS Training School 3: Experimental techniques for acoustic porous materials and metamaterials. Le Mans, France.

Jarosinska, D., Héroux, M. E., Wilkhu, P., Creswick, J., Verbeek, J., Wothge, J., \& Paunovic, E. (2018). Development of the who environmental noise guidelines for the european region: An introduction. International Journal of Environmental Research and Public Health, 15(4), 813:1-813:7.

Jimeno, B., Hau, M., \& Verhulst, S. (2018). Corticosterone levels reflect variation in metabolic rate, independent of stress. Scientific Reports, 8, 13020:1-13020:8.

Jourdan, C. (2013). Subjective geography. Retrieved 10/03/2019, from http://www .geographiesubjective.org

Kardous, C. A., \& Shaw, P. B. (2015, July). Do sound meter apps measure noise levels accurately? Sound and Vibrations, 10-13.

Kardous, C. A., \& Shaw, P. B. (2016, October). Evaluation of smartphone sound measurement applications (apps) using external microphones - A follow-up study. The Journal of the Acoustical Society of America, 140(4), 10-13.

Kleist, N. J., Guralnick, R. P., Cruz, A., Lowry, C. A., \& Francis, C. D. (2018). Chronic anthropogenic noise disrupts glucocorticoid signaling and has multiple effects on fitness in an avian community. Proceedings of the National Academy of Sciences, 115(4), E648-E657.

Kornprobst, H.-J., Manvell, D., Cook, P., \& Gigant, A. (2014). Acoustics - soundscape - part 1: Definition and conceptual framework. International Organization for Standardization, $1(1), 4$.

Kumar, S., Tansley-Hancock, O., Sedley, W., Winston, J. S., Callaghan, M. F., Allen, M., ... Griffiths, T. D. (2017). The brain basis for misophonia. Current Biology, 27(4), 527-533.

Lane, N. D., Miluzzo, E., Lu, H., Peebles, D., Choudhury, T., \& Campbell, A. T. (2010). A survey of mobile phone sensing. IEEE Communications Magazine, 10.

Lefevre, B., \& Issarny, V. (2018, June). Matching technological \& societal innovations: The social design of a mobile collaborative app for urban noise monitoring. In Proceedings of IEEE International Conference on Smart Computing (SMARTCOMP) (pp. 33-40). Taormina, Italy.

Longo, A., Zappatore, M., Bochicchio, M., \& Navathe, S. B. (2017, October). Crowd-sourced data collection for urban monitoring via mobile sensors. ACM Transactions on Internet Technology, 18(1), 5:1-5:21.

Maisonneuve, N., Stevens, M., \& Ochab, B. (2016). Participatory noise pollution monitoring using mobile phones. Information Polity, 15, 51-71.

Manwar, V. D., Mandal, B. B., \& Pal, A. K. (2016). Environmental propagation of noise 
in mines and nearby villages: A study through noise mapping. Noise and Health, 18(83), $185-193$.

Marry, S. (2011). Modèle expérimental d'évaluation d'espaces publics par les citadins à travers la perception spatiale et sonore. In RIDAD (Rencontres Interdisciplinaires Doctorales sur l'Aménagement Durable) 2011. Vaulx-en-Velin, France.

Meng, Q., \& Kang, J. (2016). Effect of sound-related activities on human behaviours and acoustic comfort in urban open spaces. Science of the Total Environment, 573, 481-493.

Miège, B. (2007). Les Tic entre innovation technique et ancrage social. Presses Universitaires de Grenoble.

915 Munzel, T., Gori, T., Babisch, W., \& Basner, M. (2014). Cardiovascular effects of environmental noise exposure. European Heart Journal, 35(13), 829-836.

Nabi, G., McLaughlin, R. W., Hao, Y., Wang, K., Zeng, X., Khan, S., \& Wang, D. (2018, July). The possible effects of anthropogenic acoustic pollution on marine mammals' reproduction: an emerging threat to animal extinction. Environmental Science and Pollution Research, $25(20), 19338-19345$.

Noureen, J., \& Asif, M. (2017). Crowdsensing: Socio-technical challenges and opportunities. (IJACSA) International Journal of Advanced Computer Science and Applications, 8(3), 363-369.

Official Journal of the European Communities. (2002). Directive 2002/49/EC of the European Parliament and of the Council of 25 June 2002 relating to the assessment and management of environmental noise. Retrieved 10/03/2019, from https://eur-lex.europa.eu/legal -content/EN/TXT/PDF/?uri=CELEX : 32002L0049\&from=EN

Olmedo, E. (2015). Représenter l'espace vécu. les enjeux d'une cartographie émotionnelle en géographie. Seminar Géographie des Émotions, ENS-Ulm.

930 Picaut, J., Can, A., Ardouin, J., Crépeaux, P., Dhorne, T., Écotière, D., .. Paboeuf, M. (2017). Characterization of urban sound environments using a comprehensive approach combining open data, measurements, and modeling. The Journal of the Acoustical Society of America, 141(5), 3808-3808.

Raap, T., Pinxten, R., Casasole, G., Dehnhard, N., \& Eens, M. (2017). Ambient anthropogenic noise but not light is associated with the ecophysiology of free-living songbird nestlings. Scientific Reports, 7, 2754:1-2754:8.

Rosa, P., \& Koper, N. (2018). Integrating multiple disciplines to understand effects of anthropogenic noise on animal communication. Ecosphere, 9(2), e02127.

Sanchez, G. M. E., Alves, S., \& Botteldooren, D. (2018). Urban sound planning: An essential component in urbanism and landscape architecture. In Handbook of research on perceptiondriven approaches to urban assessment and design (pp. 1-22).

Seixas, N., Daniell, W., Neitzel, R., Sheppard, L., \& Davies, H. (2008). Comparison of Perceived and Quantitative Measures of Occupational Noise Exposure. The Annals of Occupational Hygiene, 53(1), 41-54.

Shannon, G., McKenna, M. F., Angeloni, L. M., Crooks, K. R., Fristrup, K. M., Brown, E., ... Wittemyer, G. (2015). A synthesis of two decades of research documenting the effects of noise on wildlife. Biological Reviews, 91(4), 982-1005.

Shield, B., \& Dockrell, J. (2008). The effects of environmental and classroom noise on the academic attainments of primary school children. The Journal of the Acoustical Society of America, 123(1), 133-144.

Shield, B. M., \& Dockrell, J. E. (2003). The effects of noise on children at school: A review. J. Building Acoustics, 10(2), 97-106.

Stansfeld, S. A., \& Shipley, M. (2015). Noise sensitivity and future risk of illness and mortality. Science of the Total Environment, 520, 114-119.

Statistics, G. (2018). Number of mobile app downloads worldwide in 2017, 2018 and 2022 (in billions). Retrieved 10/03/2019, from https://www.statista.com/statistics/271644/ worldwide-free-and-paid-mobile-app-store-downloads/

The Centre for Strategy \& Evaluation Services and ACCON. (2016). Evaluation of Directive 2002/49/EC relating to the assessment and management of Environmental 
Noise. Retrieved 10/03/2019, from http://ec.europa.eu/environment/noise/pdf/ summary_study_evaluation_directive_environmental_noise_en.pdf

Troïanowski, M., Mondy, N., Dumet, A., Arcanjo, C., \& Lengagne, T. (2017). Effects of traffic noise on tree frog stress levels, immunity, and color signaling. Conservation Biology, 31 (5), $1132-1140$

Unit, T. S. C. (2016). Links between noise and air pollution and socioeconomic status. indepth report 13 (Vol. 13; Tech. Rep.). University of the West of England, Bristol: Science for Environment Policy, European Commission, DG Environment.

Unit, T. S. C. (2017). Noise abatement approaches. future brief 17 (Vol. 17; Tech. Rep.). University of the West of England, Bristol: Science for Environment Policy, European Commission, DG Environment.

Ventura, R., Mallet, V., \& Issarny, V. (2018). Assimilation of mobile phone measurements for noise mapping of a neighborhood. The Journal of the Acoustical Society of America, $144(3), 1279-1292$.

Ventura, R., Mallet, V., Issarny, V., Raverdy, P.-G., \& Rebhi, F. (2017). Evaluation and calibration of mobile phones for noise monitoring application. Journal of the Acoustical Society of America, 142(5), 3084-3093.

WHO. (2018). Environmental Noise Guidelines for the European Region (Tech. Rep.). World health Organization. Retrieved from http://www.euro.who.int/en/ health-topics/environment-and-health/noise/publications/2018/environmental -noise-guidelines-for-the-european-region-2018

Willen, R. M., Mutwill, A., MacDonald, L. J., Schiml, P. A., \& Hennessy, M. B. (2017). Factors determining the effects of human interaction on the cortisol levels of shelter dogs. Applied Animal Behaviour Science, 186, 41-48.

Zannin, P. H. T., Calixto, A., Diniz, F. B., \& Ferreira, J. A. C. (2003). A survey of urban noise annoyance in a large brazilian city: the importance of a subjective analysis in conjunction with an objective analysis. Environmental Impact Assessment Review, 23(2), 245-255.

Zhang, X., Ba, M., Kang, J., \& Meng, Q. (2018). Effect of soundscape dimensions on acoustic comfort in urban open public spaces. Applied Acoustics, 133, 73-81.

Zwicker, E., \& Fastl, H. (2007). Psychoacoustics: Facts and models. Springer-Verlag. 